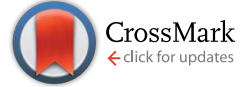

Cite this: RSC Adv., 2015, 5, 8114

Received 21st November 2014 Accepted 22nd December 2014

DOI: 10.1039/c4ra14967j

www.rsc.org/advances

\title{
Microfluidic generation of monodisperse ultra-low interfacial tension oil droplets in water $\dagger$
}

\author{
Guido Bolognesi, ${ }^{a}$ Alex Hargreaves, ${ }^{b}$ Andrew D. Ward, ${ }^{c}$ Andrew K. Kirby, ${ }^{b}$ \\ Colin D. Bain ${ }^{\mathrm{b}}$ and Oscar Ces*a
}

\begin{abstract}
We present a novel microfluidic approach for the generation of monodisperse oil droplets in water with interfacial tensions of the order of $1 \mu \mathrm{N} \mathrm{m}^{-1}$. Using an oil-in-water emulsion containing the surfactant aerosol OT, heptane, water and sodium chloride under conditions close to the microemulsion phase transition, we actively controlled the surface tension at the liquid-liquid interface within the microfluidic device in order to produce monodisperse droplets. These droplets exhibited high levels of stability with respect to rupture and coalescence rates. Confirmation that the resultant emulsions were in the ultralow tension regime was determined using real space detection of thermally-induced capillary waves at the droplet interface.
\end{abstract}

\section{Introduction}

Droplet-based microfluidics underpins the generation, transport and manipulation of femto- to picoliter sized droplets dispersed in a continuous phase. ${ }^{\mathbf{1}}$ High precision, reproducibility, the potential for ultra-high throughput and the capability to compartmentalize biological and chemical reactions within individual droplets are some of the advantages that droplet-based microfluidics offers with respect to macroscopic emulsification approaches.

The formation of droplets within microfluidic devices is generally the result of a spontaneous process where the flow viscous stresses are balanced by the interfacial tension at the liquid-liquid interface. ${ }^{2}$ However, at low and ultra-low surface tensions (namely, below $0.1 \mathrm{mN} \mathrm{m}^{-1}$ ), microfluidic droplet generation is more challenging as the growth rate of interfacial instabilities induced by the capillary forces, which drive breakup processes, are extremely low. When the capillary breakup time becomes much larger than the characteristic flow time, the effects of capillary instabilities become negligible on the time scale of thread formation and a stable jet is formed. ${ }^{3}$ Despite these technical difficulties, oil droplets in water having ultralow interfacial tensions (ULIFT) within a microfluidic environment support an ever growing number of exciting applications. As the surface tension reaches values as low as $1 \mu \mathrm{N} \mathrm{m}^{-1}$, optical fields ${ }^{4}$ can be used to sculpt oil droplets into more complex shapes,

a'Department of Chemistry, Imperial College London, London, UK. E-mail: o.ces@ imperial.ac.uk

${ }^{b}$ Department of Chemistry, Durham University, Durham, UK

${ }^{c}$ Central Laser Facility, STFC, Harwell Oxford, UK

$\dagger$ Electronic supplementary information (ESI) available: Video of a micrometer-sized oil droplet in water near the microemulsion phase transition showing thermal capillary waves at the droplet interface. See DOI: $10.1039 / \mathrm{c} 4 \mathrm{ra} 14967 \mathrm{j}$ possibly leading to new approaches for the synthesis of asymmetric solid particles with user-defined shapes. ${ }^{5}$ Additionally, in the ULIFT regime when a single droplet is separated into two droplets by two optical traps, the two droplets remain connected by a stable thread of oil with a typical diameter of less than a hundred nanometers. ${ }^{6}$ This phenomenon opens the way to the generation of complex nanofluidic networks created and controlled by light. ${ }^{7}$

In recent years, microfluidic approaches have been introduced for manufacturing ULIFT droplets by using aqueous two phase systems (ATPS). ${ }^{3}$ In such systems, droplets of one polymer solution are dispersed in another immiscible polymer solution. The water is the continuous component in both phases and the resulting interfacial tension is ultra-low. In order to perturb an otherwise stable jet and to promote droplet formation via the Rayleigh-Plateau instability, the aqueous two-phase interface can be destabilised by mechanically vibrating either the chip $^{8}$ or the soft tubing carrying the dispersed phase. ${ }^{9}$ For specific systems, electrostatic forces can also be effectively used to generate a monodisperse population. ${ }^{10}$ Interfaces with ultralow interfacial tension can also be generated with oil-water systems by adding surfactants in both phases. Similarly, long and stable jets can form ${ }^{\mathbf{1 1}}$ and mechanical vibration sources have been used to promote droplet breakup. ${ }^{12}$ However, there are a number of differences between the oil-water systems and ATPS. More specifically, the growth rates of capillary instabilities for jets formed with Newtonian oil-water phases were found to agree with the theoretical prediction for confined threads in microchannels. ${ }^{12,13}$ Conversely, the jets formed with ATPS exhibited instability growths more than an order of magnitude slower than the Newtonian counterpart under similar conditions of flows, fluid properties and degree of confinement. ${ }^{12}$ That makes the generation of ULIFT droplets more difficult in ATPS than oil-water systems. 
Additionally, for an oil-water interface the interfacial tension can enter the ultra-low regime only if enough surfactant molecules have been adsorbed at the interface. If the droplet production rate is fast enough that surfactant cannot equilibrate at the interface, common microfluidic techniques can be used for droplet generation. Indeed the dynamic surface tension is not ultra-low and the jet can spontaneously break up under the Rayleigh-Plateau instability with no need of external perturbation sources such as vibrating piezoelectric actuators or electric field generators. That has important advantages such as easier design and simpler fabrication methods for the microfluidic system. Such a strategy has been successfully used for the microfluidic generation of ULIFT water droplets in oil ${ }^{14}$ with a flow focusing junction. However, Hashimoto et $a \mathbf{l}^{\mathbf{1 4}}$ showed that once the dynamic surface tension reached its equilibrium value, spatial confinement and variation of the channel width (such as expansions and contractions) induced extreme deformation and sheardriven instabilities on droplets, thereby resulting in highly polydisperse emulsions. More specifically, the onset of RayleighPlateau instabilities in the stretched droplets promoted the formation of smaller droplets, whose final size depended on the flow conditions, ${ }^{15}$ the degree of confinement ${ }^{16}$ as well as the viscosity ratio. ${ }^{17}$ Shear-driven instabilities were instead responsible for the break up of the droplets trailing edge into daughter droplets, whose typical size was at least one order of magnitude smaller than the channel depth. In order to overcome these instabilities, emulsion could be produced at low and moderate surfactant concentrations in the continuous phase, so that the equilibrium interfacial tension would not be ultra-low and droplets would remain stable against shear-induced rupture and coalescence. A surfactant-rich continuous phase could be added to the emulsion in a separate step after formation, thereby reducing the interfacial tension to ultra-low values. However, the resulting monodisperse ULIFT droplets would be very difficult to handle since even low viscous stresses, as those induced by nearby walls, would trigger shear-driven instabilities at the droplet interface. ${ }^{18}$

In this paper, we present a microfluidic platform for the generation of stable monodisperse oil droplets in water whose final interfacial tension is of the order of $0.1-1 \mu \mathrm{N} \mathrm{m}^{-1}$. Our alternative method relies on a tuneable oil-water formulation whose surface tension can be actively controlled within the microfluidic environment. Our device is capable of producing ULIFT monodisperse droplets with diameters in the range of 10-20 $\mu \mathrm{m}$ and high stability against coalescence as well as rupture induced by hydrodynamic stresses. A microfluidic tool for the accurate and repeatable delivery of ULIFT droplets with precise control over composition and size offers the opportunity to improve and extend the field of applications of optical manipulation of ULIFT droplets ${ }^{6}$ as well as to investigate the fundamental chemistry and physics behind it.

\section{Methods and materials}

\subsection{The oil-water-surfactant system}

In our experiments heptane (VWR, 99\%) was used as the dispersed phase and an aqueous solution containing $\mathrm{NaCl}$ and diethylhexyl sodium sulphosuccinate (AOT) (Sigma-Aldrich,
98\%) as the continuous phase. Aerosol OT has closely matched hydrophilic and lipophilic properties ${ }^{\mathbf{1 9}}$ that permit phase transitions in emulsion formation by increasing the hydrophobic character, e.g. by addition of salt. Increasing salt concentration reverses the direction of the natural curvature of the surfactant film from oil-in-water to water-in-oil and hence produces an inversion in phase continuity. ${ }^{19}$ The transition between low and higher salt concentrations thus corresponds to a sharp decrease of the interfacial tension, which can reach values down to $0.1 \mu \mathrm{N} \mathrm{m}^{-1}$, provided the surfactant concentration exceeds the critical micelle concentration (cmc). A similar transition occurs if the salinity of the aqueous solution is kept constant and the emulsion temperature changes. For instance, at a salt concentration of $50 \mathrm{mM}$ and temperatures higher than $26^{\circ} \mathrm{C}$, AOT is hydrophilic and the surfactant only partitions into the oil phase at lower temperatures. Again, when the transition occurs, the surface tension goes into the ULIFT regime (i.e. $\sim 1 \mu \mathrm{N} \mathrm{m}^{-1}$ ). The temperature corresponding to the minimum attainable interfacial tension for a given salinity is denoted as the phase inversion temperature (PIT). For AOT solutions, the PIT varies from $15{ }^{\circ} \mathrm{C}$ to $40{ }^{\circ} \mathrm{C}$ when salt concentration increases from $33 \mathrm{mM}$ to $84 \mathrm{mM}$. The relationship between PIT and salinity as well as the dependency of interfacial tension on salt concentration and temperature for the heptane-NaCl-water-AOT system have been characterized by Aveyard et al. ${ }^{20-22}$ through spinning droplet tensiometry.

\subsection{Microfluidic chips and image system}

Droplets were generated in a $14 \mu \mathrm{m}$ depth glass flow-focusing device with a cross-junction, ${ }^{23}$ fabricated by Dolomite Microfluidics via standard photo-lithography and glass etching techniques. The cross-junction consisted of four channels $17 \mu \mathrm{m}$ in width and $135 \mu \mathrm{m}$ in length which joined at right angles. The cross-junction was connected to $500 \mu \mathrm{m}$ width inlet and outlet channels via expansion and contractions (see Fig. 1b). Hereafter this chip is referred to as the flow focusing junction (FFJ) chip. Syringe pumps (WPI-Aladdin2220) were used to supply the dispersed phase in the central channel and the continuous phase in the side channels. Measurements were performed at least five minutes after changing the flow rates of the two phases to ensure the system stability. The FFJ chip was also fitted with on-chip temperature control (see Fig. 1c), consisting of a resistance thermometer (PT100 Class A) and a Peltier cell (Ferrotec) attached to the bottom and top external walls of the chip, respectively. The Peltier cell had a central $5 \mathrm{~mm}$ diameter hole to allow optical access to the cross-junction. The cell was driven by a proportional-integral-derivative (PID) control unit (Electron Dynamics Ltd.), which allowed for temperature control with a precision of $0.1{ }^{\circ} \mathrm{C}$. In order to allow the system to reach thermal equilibrium, measurements began at least three minutes after the chip temperature had been set to a new value. In some experiments, the temperature control unit was de-activated so that droplet generation could be performed under laboratory ambient conditions. 
a)

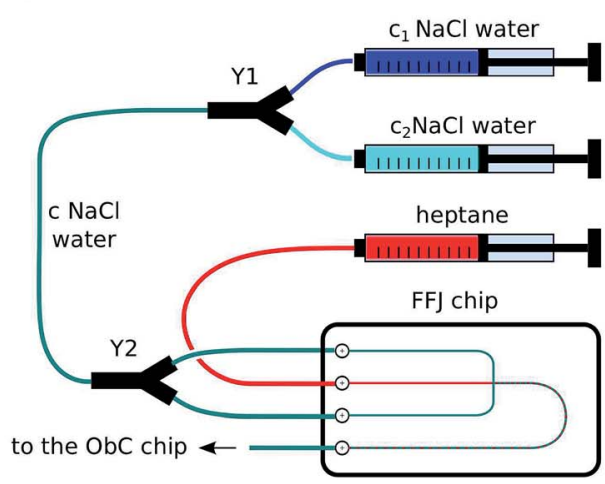

b)

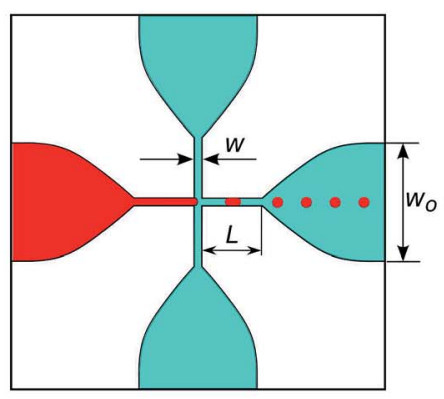

C)

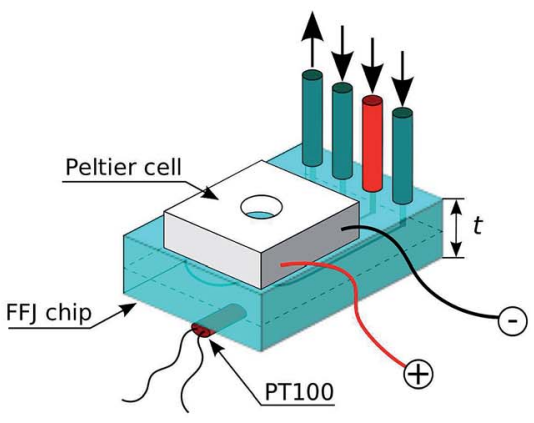

Fig. 1 (a) Schematic view of the microfluidic platform. Two syringe pumps were used to mix two aqueous solutions with concentrations $c_{1}$ and $c_{2} \mathrm{NaCl}$. The mixed solution with final salinity $\mathrm{c} \mathrm{NaCl}$ supplied the side channels of the flow focusing junction (FFJ) chip. The central channel of the chip was fed with heptane by a third syringe pump. (b) Scheme of the cross-junction where heptane droplets were formed. The dimensions of the channels are the following: $w_{\circ}=500 \mu \mathrm{m}, L=135 \mu \mathrm{m}, w=17 \mu \mathrm{m}$. (c) Three dimensional schematic view of the on-chip temperature control system. The channels were located in the middle plane (dashed lines) of the FFJ glass slab, whose total thickness was $t=4 \mathrm{~mm}$.

After formation, droplets were transferred into a separate device designed and fabricated for droplet storage and characterization purposes. The device was made of two microscope slides separated by a PDMS layer, the fabrication protocol being reported elsewhere. ${ }^{24}$ We refer to that device as the observation chamber (ObC) chip.

Images of droplets within both the FFJ and ObC chips were captured with an Olympus IX81 inverted microscope fitted with a CCD camera (Q-Imaging Retiga EXi fast). Image postprocessing for droplet sizing and characterization was performed with custom Java macros implemented in Image and Python code.

\section{The microfluidic platform}

The heptane-water emulsion containing AOT and $\mathrm{NaCl}$ reaches the ULIFT regime when the system is very close to the microemulsion phase transition. Such a condition can be obtained by finely tuning the salinity level and the temperature of the emulsion. We thus devised a microfluidic system where both those parameters could be controlled. The final salinity of the continuous phase was obtained by mixing two aqueous solutions with different salt concentrations. Two syringes (see Fig. 1a) were charged with $c_{1}$ and $c_{2} \mathrm{mM} \mathrm{NaCl}$ aqueous solutions plus $2 \mathrm{mM}$ AOT. The liquids from the two syringes joined together in a Y junction (Y1) and fully mixed by flowing in a $250 \mu \mathrm{m}$ diameter FEP tubing. Denoting the flow rates in the two syringes as $Q_{c_{1}}$ and $Q_{c_{2}}$, the final salt concentration of the mixed phase can be expressed as

$$
c=\frac{c_{1} Q_{c_{1}}+c_{2} Q_{c_{2}}}{Q_{c_{1}}+Q_{c_{2}}}
$$

After mixing, the flow split at the junction Y2 and entered the side channels of the FFJ chip. Assuming a relative error of $\delta c_{i} / c_{i}$ $=0.2 \%$ (for $i=1,2$ ) for the salinity of the aqueous solutions and $\delta Q_{c_{i}} / Q_{c_{i}}=1 \%$ (for $i=1,2$ ) for the flow rates of the syringe pumps, we can estimate that the accuracy on the salt concentration of the mixed phase is about $\delta c / c=0.3 \%$. For a $50 \mathrm{mM}$ $\mathrm{NaCl}$ aqueous solution, that means an approximate error of $0.2 \mathrm{mM}$ in the final salt concentration.

The chip temperature was set through a thermal control unit. Denoting the thermal diffusivity of any liquid phase as $\alpha_{\mathrm{T}}$, the liquid reaches thermal equilibrium with the surrounding walls after flowing for a distance $l_{\mathrm{T}} \simeq Q / 2 \alpha_{\mathrm{T}}$, where $Q$ is the liquid flow rate. Considering the thermal diffusivity of water $\left(\alpha_{\mathrm{T}}=14.3 \times 10^{-8} \mathrm{~m}^{2} \mathrm{~s}^{-1}\right)$ and heptane $\left(\alpha_{\mathrm{T}}=8.5 \times 10^{-8} \mathrm{~m}^{2} \mathrm{~s}^{-1}\right)$ at $25{ }^{\circ} \mathrm{C}$ and a maximum flow rate $Q=20 \mu \mathrm{L} \mathrm{min}{ }^{-1}$, we obtain $l_{\mathrm{T}} \simeq 1-2 \mathrm{~mm}$. Since the area covered by the Peltier cell is 13 $\mathrm{mm} \times 13 \mathrm{~mm}$, it can be assumed that at the cross-junction both phases are at thermal equilibrium with the channel walls. By performing stationary finite element analysis in Comsol Multiphysics (COMSOL Ltd.), the temperature field in the FFJ glass slab was determined assuming that heat enters the system from the Peltier module and leaves it through the external walls by natural convection. With a typical room temperature of $23{ }^{\circ} \mathrm{C}$, for an average chip temperature of $30{ }^{\circ} \mathrm{C}$ the standard deviation of the temperature field in the volume of the FFJ glass slab under by the Peltier unit is about $0.2{ }^{\circ} \mathrm{C}$. That value can be considered as a good estimate for the precision of the emulsion temperature measurements.

\section{Results and discussion}

Droplets cannot readily be generated in the ULIFT regime because the effects of capillary forces, which drive the interfacial instability leading to the breakup of the dispersed phase stream, are dramatically slowed down. Consequently, for surface tension lower than a critical value $\gamma_{c}$ (see Appendix) droplets do not form at the cross-junction and parallel flows of the two phases are obtained instead. If, however, the time scale for interface generation is less than the characteristic diffusion time for the surfactant to the interface, the equilibrium surface coverage is not reached on the time scale of droplet formation. ${ }^{25}$ 
Under these conditions, the dynamic surface tension $\gamma_{d}$ assumes values between the surface tension at equilibrium $\gamma_{\mathrm{e}}$ and the surface tension of a clean surfactant-free interface $\gamma_{0}$, depending on the device geometry, the flow rates and the type and concentration of surfactant. ${ }^{25}$ For $\gamma_{d}$ typically higher than $\gamma_{c}$, it is hence possible to produce monodisperse droplets immediately at the junction whereas the droplet equilibrium surface tension still lies in the ULIFT regime. For typical surfactant concentrations in the millimolar range, the diffusion timescale is of the order of milliseconds. After formation, the droplets go through the junction and enter the wider output channel, where the diffusive and convective transport of surfactant molecules to the interface ${ }^{25}$ causes the surface tension $\gamma_{\mathrm{d}}$ to drop to ultra-low values on a time scale of tens of milliseconds after generation. For that reason, ULIFT droplets are unstable and they can easily tear apart due to the high viscous stresses downstream of the junction, yielding a highly polydisperse emulsion. ${ }^{\mathbf{1 4}}$

The most effective method to produce and manipulate monodisperse ULIFT droplets is to control the equilibrium surface tension according to the operation required. For droplet generation and transport, $\gamma_{\mathrm{e}}$ has to be higher than $\gamma_{\mathrm{c}}$ and the capillary number $\mathrm{Ca}$ lower than a critical value $\mathrm{Ca}_{\mathrm{c}}$ whereas for droplet storage, manipulation and optical deformation $\gamma_{\mathrm{e}}$ can be lowered down to the ULIFT regime. Such control is quite difficult to implement when $\gamma_{\mathrm{e}}$ mainly depends on the concentration of surfactant in either phases, as it occurs for the oil-water-surfactant systems typically used in droplet microfluidics. Moreover, in those systems where surfactant concentration exceeds the $\mathrm{cmc}, \gamma_{\mathrm{e}}$ reaches its minimum value and it no longer depends on the amount of surfactant.

On the contrary, for the heptane-brine-AOT system at a surfactant concentration higher than the cmc, $\gamma_{\mathrm{e}}$ strongly depends on salinity and temperature. By controlling these two parameters, the equilibrium surface tension can be independently tuned in the FFJ chip for droplet generation and in the ObC chip for droplet storage and characterization. In the following sections, we separately assess the effects of salinity and temperature on droplet formation and we show how this microfluidic platform can be used to generate and store monodisperse ULIFT droplets.

\subsection{Effect of salinity on droplet formation}

According to the literature,$^{20}$ the equilibrium surface tension of the heptane-water-AOT-NaCl system at $25{ }^{\circ} \mathrm{C}$ reaches values below $10 \mu \mathrm{N} \mathrm{m}^{-1}$ for salt concentrations in the range of $38 \mathrm{mM}-58 \mathrm{mM}$. To assess the effects of salinity on droplet formation, the FFJ chip temperature was fixed and the emulsion salinity was varied in the range corresponding to the lowest surface tensions. The continuous phase syringes were filled with aqueous solution at salt concentrations $c_{1}=0 \mathrm{mM}$ and $c_{2}=100 \mathrm{mM}$. The corresponding flow rates $Q_{c_{1}}$ and $Q_{c_{2}}$ were

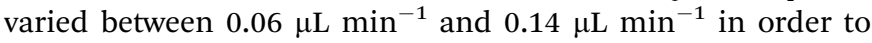
tune the final salinity of the emulsion. The total water flow rate $Q_{\mathrm{c}}=Q_{c_{1}}+Q_{c_{2}}$ and oil flow rate $Q_{\mathrm{d}}$ were fixed to $0.2 \mu \mathrm{L} \mathrm{min}{ }^{-1}$ and $0.05 \mu \mathrm{L} \min ^{-1}$, respectively. The droplet generation was performed under laboratory ambient conditions. The lab temperature was monitored through the on-chip resistance thermometer and it was constant at $23.6{ }^{\circ} \mathrm{C}$ for all experiments.

Under these conditions, $\gamma_{\mathrm{e}}$ depends strongly on the concentration of $\mathrm{NaCl}$ and its value can be qualitatively assessed through the deformations droplets underwent in the expanding output channel. Fig. 2 shows the generated droplets flowing outside of the junction for salinity levels between $35 \mathrm{mM}$ and $60 \mathrm{mM}$. By interpolating the experimental data available in the literature, ${ }^{20}$ we could estimate that the equilibrium surface tensions $\gamma_{\mathrm{e}}$ varied in the range of 1-22 $\mu \mathrm{N} \mathrm{m}^{-1}$ (see labels in Fig. 2). As droplets left the junction, the viscous stresses exerted by the continuous phase flow in the expanding output channel deformed the droplets.

The continuous phase flow was quite complex for the examined channel geometry. It had an irrotational component induced by the increasing width of the channel as well as a rotational component induced by the over-confinement of the droplets, whose undeformed diameter $d_{0}$ was typically larger than the channel depth $h$. Moreover, the simultaneous presence of several droplets in the output channel affected the flow itself.

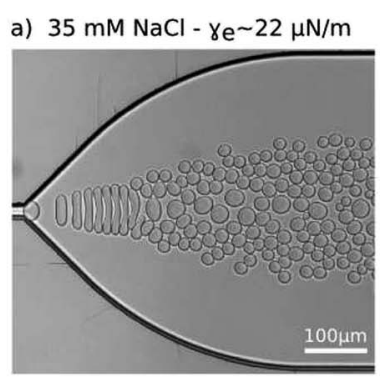

c) $45 \mathrm{mM} \mathrm{NaCl}-\gamma \mathrm{e} \sim 1.0 \mu \mathrm{N} / \mathrm{m}$

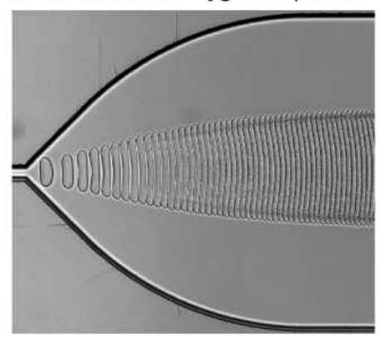

e) $55 \mathrm{mM} \mathrm{NaCl}-\gamma \mathrm{e} \sim 4.0 \mu \mathrm{N} / \mathrm{m}$

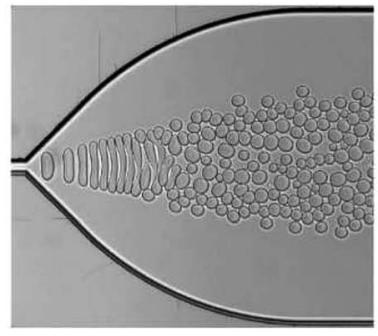

b) $40 \mathrm{mM} \mathrm{NaCl}-\gamma \mathrm{e} \sim 9.0 \mu \mathrm{N} / \mathrm{m}$

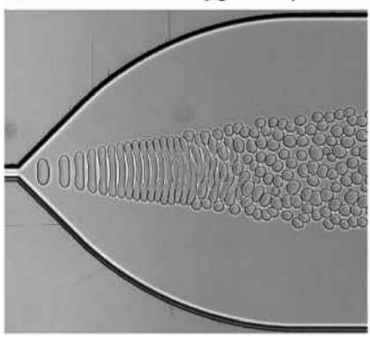

d) $50 \mathrm{mM} \mathrm{NaCl}-\gamma \mathrm{e} \sim 1.5 \mu \mathrm{N} / \mathrm{m}$

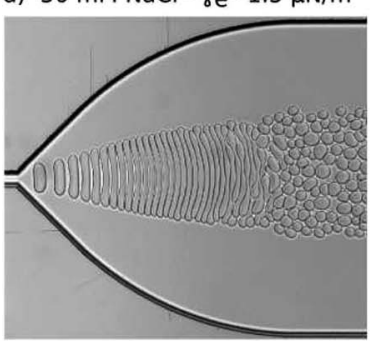

f) $60 \mathrm{mM} \mathrm{NaCl}-\gamma \mathrm{e} \sim 7.5 \mu \mathrm{N} / \mathrm{m}$

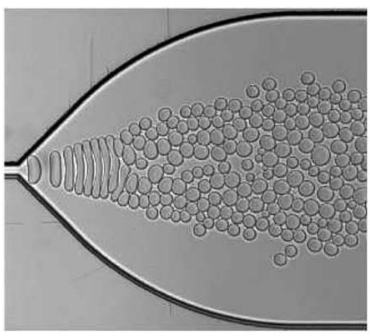

Fig. 2 Outside of the junction, the droplets were stretched by the surrounding flow and assumed very elongated shapes. At the top of each panel, the salt concentration and the estimated corresponding equilibrium surface tension are reported. The typical production rate is about $200 \mathrm{~Hz}$. The surface tension were interpolated from data in ref. 20 . 
As drops slowed down, the separation distance between them was reduced and the fluid was squeezed out from the region between the drops, thereby increasing the total strain rate applied to the drops. ${ }^{14}$ Finally, the viscous stresses exerted on individual droplets was not constant in time since as the droplet abandoned the expanding section of the output channel the corresponding extensional component of the flow ceased.

Generally, if the capillary number exceeds a critical value $\mathrm{Ca}_{\mathrm{c}}$, no equilibrium droplet shape exists in order to balance the viscous and capillary forces and the flow keeps deforming the droplets until Rayleigh-Plateau instabilities prevail and droplet breakup occurs. Droplet breakup of confined droplets for specific flows, such as simple shear and extensional, have been investigated in the literature.$^{\mathbf{1 8 , 2 6}}$ For instance, for viscosity ratio $\lambda<1$ and simple shear flow, ${ }^{26,27}$ spatial confinement is expected to stabilize the droplets by increasing the value of $\mathrm{Ca}_{c}$ with respect to the case of unbounded flow. For viscosity ratio $\lambda=1$ and over-confinement $\left(d_{0}>h\right)$, extreme droplet deformations have also been reported, ${ }^{28}$ the final droplet length before rupture being up to 10 times the undeformed droplet radius. Even though the geometry of our droplet generation device does not allow to easily estimate the character and the intensity of the viscous stresses, and, hence, of the $\mathrm{Ca}_{\mathrm{c}}$, our experimental data showed that for the examined flow rates droplets did not break up in the output channel when the equilibrium surface tension was higher than $0.1 \mathrm{mN} \mathrm{m}^{-1}$. Moreover, at constant temperature (hence, constant liquid viscosity) and constant liquid flow rates, the viscous stresses are expected to be the same for all experiments. Consequently, the Ca number was a function of the surface tension only and the deformed shape of the droplets exclusively depended on the surface tension of the oil-water interface with higher deformations corresponding to lower values of $\gamma_{\mathrm{e}}$.

In agreement with our physical description of the process, Fig. 2 shows that for most salt concentration, the degree of droplet deformation kept increasing as droplets flowed downstream up to the point that the capillary instability prevailed and the droplet broke up into two or more smaller droplets. That behaviour is very similar to the "rain" regime reported for a Hele-Shaw cell. ${ }^{\mathbf{1 4}}$ Despite the fact that droplets had very low interfacial tension, they showed high stability against coalescence, on the timescale of a few hundred milliseconds. That stability is essential for subsequent transport and storage of the droplets. The droplet deformability can be assessed through the highest ratio $D_{\max }=l / d_{0}$ reached by the droplet before rupture, $l$ being the droplet length. At $35 \mathrm{mM} \mathrm{NaCl}\left(\gamma_{\mathrm{e}} \simeq 22 \mu \mathrm{N} \mathrm{m}^{-1}\right)$, $D_{\max }$ was about 4 whereas at $45 \mathrm{mM} \mathrm{NaCl}\left(\gamma_{\mathrm{e}} \simeq 1 \mu \mathrm{N} \mathrm{m}^{-1}\right)$ it went up to 9 . In the latter case, the salt concentration was very close to the value corresponding to the phase inversion (namely, $46.5 \mathrm{mM}$ at $23.6{ }^{\circ} \mathrm{C}$ ) and the droplets remained stable to break up within the field of view of the microscope (Fig. 2c). A similar condition occurs in the "fishbone" regime for a Hele-Shaw cell. ${ }^{14}$ Such result can be explained by comparing the breakup time and the drop residence time in the field of view. We can assume that in supercritical conditions $\left(\mathrm{Ca}>\mathrm{Ca}_{\mathrm{c}}\right)$, the time of capillary breakup scales with the visco-capillary time $t_{\mathrm{vc}}=\frac{\eta_{\mathrm{c}} d_{0}}{\gamma_{\mathrm{e}}}$.
Under the same condition of temperature and flow rates, lower interfacial tensions would hence result in longer breakup time. For extremely low interfacial tensions $\left(\sim 1 \mu \mathrm{N} \mathrm{m}^{-1}\right)$, the breakup time exceeded the residence time and droplets no longer broke up within the field of view of the microscope.

To conclude, our experiments show that droplet deformability depends on water salinity and it increases with decreasing $\gamma_{\mathrm{e}}$. Such behaviour demonstrates the capability of the microfluidic platform to tune the equilibrium surface tension of the oil-water interface by controlling the salt concentration in the continuous phase.

\subsection{Effect of temperature on droplet formation}

The control over the salt concentration allowed us to bring the water-oil system very close to the microemulsion phase transition, thereby generating very low interfacial tensions. As shown in Fig. 2, that condition is not ideal for droplet generation because droplets were so deformable that viscous stresses could tear them apart, resulting in a highly polydisperse population. However, for a given salinity the equilibrium interfacial tension can still be modified through temperature. Therefore we used the on-chip temperature control unit to increase the equilibrium surface tension in the FFJ chip so that droplets no longer broke.

According to the literature, ${ }^{20}$ at $50 \mathrm{mM} \mathrm{NaCl}$ the surface tension drops below $10 \mu \mathrm{N} \mathrm{m}^{-1}$ in a temperature range of $18{ }^{\circ} \mathrm{C}$ and $29^{\circ} \mathrm{C}$. In order to assess the effect of temperature on droplet formation in the device, the system was first brought very close to the microemulsion phase transition (namely, 47.3 $\mathrm{mM} \mathrm{NaCl}$ and $24.3^{\circ} \mathrm{C}$ ) and then the temperature was gradually increased while keeping the salt concentration constant. As for the previous experiments, the total flow rate of the continuous phase was $Q_{\mathrm{c}}=0.2 \mu \mathrm{L} \min ^{-1}$ whereas the flow rate of the dispersed phase was $Q_{\mathrm{d}}=0.05 \mu \mathrm{L} \mathrm{m^{-1 }}{ }^{-}$. Under those conditions, the droplet surface tension is expected to increase with the temperature. We note that the increase in temperature does not only affect the interfacial property of the emulsion, but it also changes the bulk rheology of the liquid phases. Indeed the viscosity of heptane and water decrease by about $19 \%$ and $35 \%$, respectively, for a temperature rise from $20{ }^{\circ} \mathrm{C}$ to $40{ }^{\circ} \mathrm{C}$. The viscosity affects the fluid stresses as well as the droplet size so it is not possible to relate the droplet shape directly to the surface tension as in the previous experiments.

Fig. $3 \mathrm{a}$ and $\mathrm{b}$ show droplets at the junction exit at temperatures of $29.9{ }^{\circ} \mathrm{C}$ and $43.4{ }^{\circ} \mathrm{C}$, respectively. It is evident that droplet formation and shape were highly affected by temperature. Interpolating the data available in the literature, ${ }^{\mathbf{2 0}}$ the equilibrium surface tension at $29.9{ }^{\circ} \mathrm{C}$ was estimated to be $16 \mu \mathrm{N} \mathrm{m}^{-1}$. On the other hand, $43.4{ }^{\circ} \mathrm{C}$ is outside of the range of temperature for which surface tension measurements are available at the examined salinity. However, since that temperature is about $20{ }^{\circ} \mathrm{C}$ higher than the PIT, we can reasonably assume $\gamma_{\mathrm{e}}>0.1 \mathrm{mN} \mathrm{m}^{-1}$. As expected, the experimental results show that for a high value of $\gamma_{e}$, the capillary force successfully opposed the viscous stresses, thereby preventing droplet rupture in the outlet channel. The resulting 
a) $29.9^{\circ} \mathrm{C}-\mathrm{\gamma e} \sim 16 \mu \mathrm{N} / \mathrm{m}$

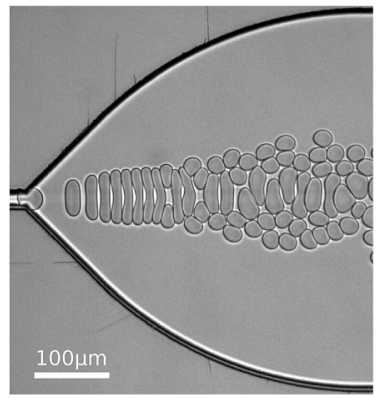

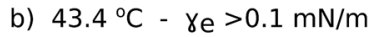

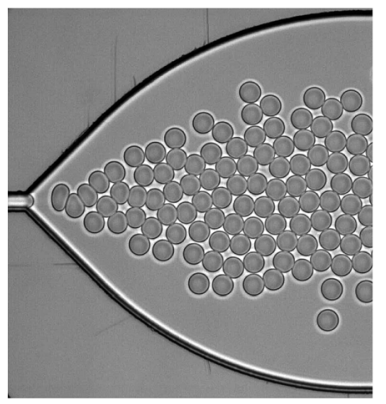

Fig. 3 Droplets at the junction exit with salt concentration of $47.3 \mathrm{mM}$ at temperatures higher than the corresponding PIT, namely $24.3^{\circ} \mathrm{C}$. The equilibrium surface tension increased with increasing temperature and for values higher than $0.1 \mathrm{mN} \mathrm{m}^{-1}$, the droplet population was monodisperse. In panel (b), the droplet diameter is $22.7 \pm 0.8 \mu \mathrm{m}$.

droplet population was monodisperse. To assess quantitatively the monodispersity of the emulsion, 180 droplets from images similar to Fig. $3 \mathrm{~b}$ were analysed, resulting in an average droplet diameter of $22.7 \mu \mathrm{m}$ with a coefficient of variation (i.e. the ratio of the standard deviation and the mean value) less than $3.5 \%$. In previous studies on droplet formation in a cross-junction device, ${ }^{29}$ it has been demonstrated that the droplet diameter scales as $\left(Q_{\mathrm{c}} / Q_{\mathrm{d}}\right)^{-a} \mathrm{Ca}^{-b}$, where $a$ and $b$ are positive constants. If we thus increase the flow rate ratio $Q_{\mathrm{c}} / Q_{\mathrm{d}}$ and the continuous phase flow rate $Q_{\mathrm{c}}$, we can generate even smaller droplets. As an

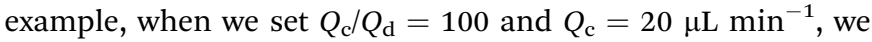
obtained droplets with an average diameter of $11 \mu \mathrm{m}$ and a coefficient of variation less than $5 \%$ (data not shown).

\subsection{Thermal capillary waves at the ULIFT droplet interface}

In order to demonstrate the capability of the microfluidic platform to produce an emulsion with such a low surface tension, we generated and stored droplets under temperature and salinity conditions corresponding to the ULIFT regime and monitored the thermally-driven perturbations at the droplet interface. It is well known that thermal motion is able to produce statistical fluctuations of the position of a two-phase interface. ${ }^{30}$ The typical amplitude of those thermally-induced capillary waves scales as $\sqrt{k_{\mathrm{B}} T / \gamma}$, where $k_{\mathrm{B}}$ is the Boltzmann constant and $T$ the absolute temperature. For the most common water-oil-surfactant systems adopted in droplets microfluidics, the surface tension is of the order of 1 to $10 \mathrm{mN} \mathrm{m}^{-1}$, which sets the wave amplitude in the range of $1 \mathrm{~nm}$. Such a small perturbation can only be detected via light and X-ray scattering techniques and, hence, the interface of a droplet appears to be smooth down to the molecular length scale. On the other hand, if the surface tension is lowered down to $0.1-1 \mu \mathrm{N} \mathrm{m}^{-1}$, the interface roughness increases up to $50-200 \mathrm{~nm}$, and the thermal capillary waves can be observed in real space through standard optical microscopy. ${ }^{31}$

Droplet were generated with a salt concentration whose corresponding PIT was equal to the lab temperature. Under such conditions, the equilibrium surface tension is expected to be very close to the minimum value, which is less than
$1 \mu \mathrm{N} \mathrm{m}^{-1}$. Droplet production and transport to the ObC chip was performed at temperatures higher than $40{ }^{\circ} \mathrm{C}$, so that viscous stresses did not break the droplets and the emulsion remained monodisperse. As the droplets reached the ObC chip, the flow was stopped and the droplets, at rest, were allowed to equilibrate at the lab temperature. Alternatively, droplets could also be produced at lab temperature but with high salinity levels to satisfy the condition $\gamma_{\mathrm{e}}>\gamma_{\mathrm{c}}$ and the ObC chip temperature could be adjusted with a heating device to let the emulsion enter the ULIFT regime. As the emulsion temperature approached the PIT, the droplet interfaces began to fluctuate under the effect of thermal motion. Fig. 4 shows the thermal capillary waves at the interface of a $15 \mu \mathrm{m}$ diameter droplet (Video available in the ESI $\dagger$ ). Tracking the motion of the droplet interface we measured a root mean squared displacement of approximately $\Delta s=110 \pm 50 \mathrm{~nm}$ (see Fig. 4e), which according to the scale law $\Delta s \simeq\left(k_{\mathrm{B}} T / \gamma\right)^{1 / 2}$ is consistent with a surface a)

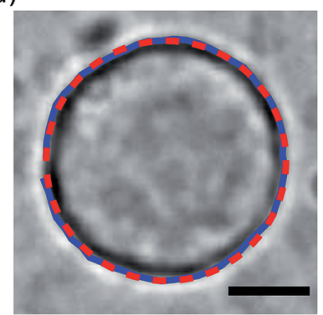

c)

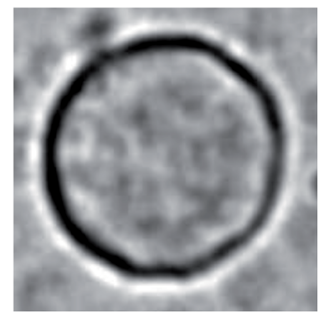

e) b)

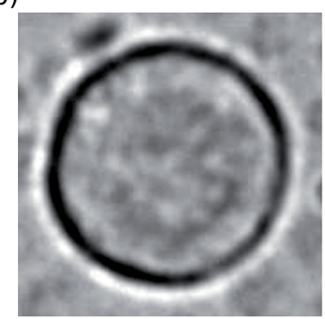

d)

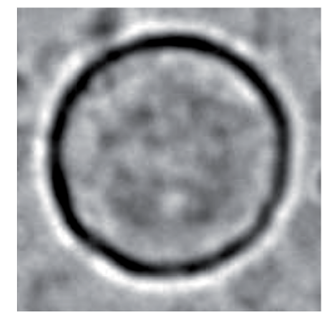

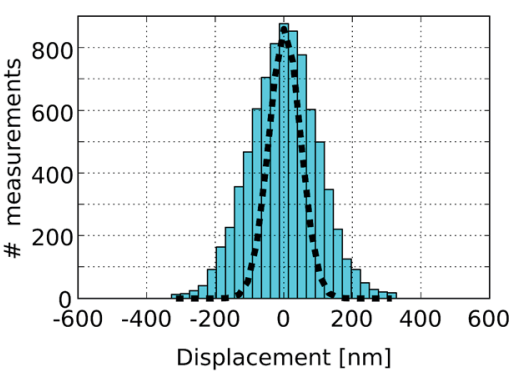

Fig. 4 Thermal capillary waves at the interface of a droplet near the microemulsion phase transition $\left(44.5 \mathrm{mM} \mathrm{NaCl}\right.$ at $22.2^{\circ} \mathrm{C}$ ). (a-d): four consecutive frames extracted from a $10 \mathrm{fps}$ frame rate video. The full video is available in the (ESI $\dagger)$. The scale bar is $5 \mu \mathrm{m}$. The solid line in panel (a) shows the actual interface position whereas the dashed lines is the corresponding best fit circle. (e) Histogram of the droplet interface displacements, which are computed as the radial distance between the actual interface and the best fit circle. The dashed line is the corresponding histogram for a rigid droplet, whose salinity and temperature conditions are far from the microemulsion phase transition. 
tension lower than $1 \mu \mathrm{N} \mathrm{m}^{-1}$, as expected. The root mean square displacement of the interface of a rigid droplet (namely, $50 \mathrm{~nm}$ ) is considered as a good estimate for the accuracy of the droplet interface tracking method. The comparison between the histograms of the interface displacements for a deformable droplet and a rigid one (see Fig. 4e) shows that the adopted bright-field interface tracking method is accurate enough to discriminate between ULIFT and non-ULIFT droplets. Higher accuracy for the interface tracking can be obtained by using alternative optical methods as, for instance, fluorescence microscopy. ${ }^{31}$

\section{Conclusions}

In this paper we present a novel approach for the stable generation of monodisperse ULIFT oil droplets in water. We have devised and successfully tested a microfluidic platform, which allowed us to tune the surface tension of the liquidliquid interface by accurate control of the salinity and temperature. As a consequence of that, droplets could be generated with common microfluidic techniques, such as the use of flowfocusing device, with no need of external perturbation sources as required when ATPS are used. After formation, droplets were transferred in a separate chip for storage and characterization purposes. The aqueous phase was pumped from two syringes filled with solutions having different salt concentrations. The flow rate ratio of the syringes determined the final salinity of the emulsion. On-chip temperature control units were adopted to adjust the emulsion temperature.

Droplet formation in microfluidic devices is challenging when the interfacial tension is in the ULIFT regimes. The growths of interfacial disturbances, which drive the capillary breakup, are extremely delayed and the dispersed phase can form long and stable jets. Using the theory of absolute and convective instabilities for confined jets, we determined a critical value for the surface tension of about $0.1 \mathrm{mN} \mathrm{m}^{-1}$, above which no jet is stable and droplets form immediately at the cross-junction. We showed that droplets can be generated even for equilibrium surface tensions in the ULIFT regimes, but only if the production rate is fast enough that surfactant cannot equilibrate at the interface. However, after formation the dynamic surface tensions quickly drop to the equilibrium ultralow value and the resulting capillary forces cannot compete against the viscous stresses; the droplets tear apart and the resulting emulsion is polydisperse.

For that reason, the droplet production and manipulation were performed at temperatures higher than $40{ }^{\circ} \mathrm{C}$ for saltsurfactant-water-oil formulations with PIT close to $20{ }^{\circ} \mathrm{C}$. Under those conditions, the hydrodynamic instabilities, which characterize the dynamics of ULIFT drops, ${ }^{\mathbf{1 4}}$ could be avoided and the formed droplets were highly stable with respect to rupture as well as coalescence. As droplets were collected in a separate device and brought under conditions close to the microemulsion phase transition, thermally-driven capillary waves with a typical amplitude of $100 \mathrm{~nm}$ were observed at the liquid-liquid interface, thereby proving that the emulsion had finally entered the ULIFT regime.

\section{Appendix}

At low Reynolds and Weber numbers, the droplet formation process is controlled by the balance of viscous and capillary forces. Viscous forces tend to stabilize the interfaces between the two phases whereas the capillary forces promote the growth of interfacial disturbances which eventually lead to the breakup of the dispersed phase stream. The time of breakup scales as the visco-capillary time $t_{\mathrm{vc}}=\eta_{\mathrm{c}} a / \gamma$, where $\eta_{\mathrm{c}}$ is the dynamic viscosity of the continuous phase, $a$ is the radius of the dispersed phase thread and $\gamma$ is the surface tension. At ultra-low values of surface tension, $t_{\mathrm{vc}}$ becomes much larger than the characteristic flow time and the onset and growth of the interfacial perturbations is extremely delayed. Under these conditions, the effects of capillary forces are negligible on the time scale of thread formation and a long and stable jet can form. Considering the geometry, the flow rates and the liquid properties of our microfluidic system, we now determine an estimate of the minimum value of surface tension for which jets are no longer stable and droplets break up promptly at the junction.

According to the theoretical predictions of Guillot et al., ${ }^{32}$ the disturbance growth rate for a jet of radius $r_{0}$ and viscosity $\eta_{\mathrm{d}}$, confined in a cylindrical channel of radius $R_{\mathrm{c}}$ and focused by a continuous phase stream of viscosity $\eta_{\mathrm{c}}$ is given by

$$
\omega=\frac{\gamma}{16 \eta_{\mathrm{c}} R_{\mathrm{c}}} \frac{F(x, \lambda)\left(\tilde{k}^{2}-\tilde{k}^{4}\right)}{x^{9}\left(1-\lambda^{-1}\right)-x^{5}}
$$

where $\tilde{k}=k r_{0}$ is the dimensionless wavenumber of the perturbation, $x=r_{0} / R_{\mathrm{c}}$ is the dimensionless jet radius, $\lambda=\eta_{\mathrm{d}} / \eta_{\mathrm{c}}$ is the viscosity ratio and the function $F(x, \lambda)$ is equal to $F(x, \lambda)=x^{4}(4-$ $\left.\lambda^{-1}+4 \ln x\right)+x^{6}\left(-8+4 \lambda^{-1}\right)+x^{8}\left(4-3 \lambda^{-1}-\left(4-4 \lambda^{-1}\right) \ln x\right)$. The radius of the perturbed jet is equal to $r=r_{0}+\varepsilon_{0} \exp (i k z+\omega t)$, where $r_{0}$ is the unperturbed jet radius and $\varepsilon_{0}$ is the initial perturbation amplitude. Numerical simulations have showed that eqn (2) provides accurate results also for jets flowing in square channels at low and moderate degrees of confinement (namely, $x<0.6) .{ }^{13}$ Since the junction cross-section of the chip used in our experiments is almost square, eqn (2) can be used to estimate the growth rate of the fastest disturbance by replacing the channel radius $R$ with the equivalent radius $\sqrt{w h} / 2$, where $w$ and $h$ are the junction width and depth, respectively.

In order to prove that droplets cannot be easily produced in the ULIFT regime, we now determine the jet break up time for ultra-low values of surface tension. If we assume the flow rate and viscosity of the continuous (dispersed) phase to be $Q_{\mathrm{c}}=0.2$ $\mu \mathrm{L} \min ^{-1}\left(Q_{\mathrm{d}}=0.05 \mu \mathrm{L} \min ^{-1}\right)$ and $\eta_{\mathrm{c}}=10^{-3}$ Pa s $\left(\eta_{\mathrm{d}}=0.39 \times\right.$ $10^{-3} \mathrm{~Pa} \mathrm{~S}$ ), respectively, we can predict from the Stokes equations $^{32}$ a dimensionless jet size of $x=0.3$ and from eqn (2) a maximal growth rate of $\omega^{*}=20 \mathrm{~Hz}$ at the dimensionless wavenumber $\tilde{k}^{*}=0.7$. The jet breakup time $t_{\mathrm{b}}$ can be defined as the time at which the disturbance amplitude equals the unperturbed jet radius, namely $\varepsilon_{0} \exp \left(\omega^{*} t_{\mathrm{b}}\right)=r_{0}$. If we assume an initial perturbation amplitude $\varepsilon_{0}$ of the order of $1 \mathrm{~nm}$ and a surface tension in the ULIFT regime, such as $1 \mu \mathrm{N} \mathrm{m}^{-1}$, the fastest perturbation will take about $t_{\mathrm{b}}=0.4 \mathrm{~s}$ to break up the dispersed 
phase stream. If the cross-junction channel were long enough, we could expect that in the ULIFT regime a stable jet with a total length more than 300 times the junction width would form. Under such conditions, an accurate control over the size and monodispersity of the droplet population would be extremely difficult.

The extremal velocity of the envelope of the perturbation can be written as ${ }^{32}$

$$
v_{ \pm}^{*}=\frac{\operatorname{Ca} x^{3} E(x, \lambda) \pm C_{1} F(x, \lambda)}{x^{9}\left(1-\lambda^{-1}\right)-x^{5}}
$$

where $\mathrm{Ca}$ is a Capillary number defined as $\frac{-\delta_{z} P^{0} R_{\mathrm{c}}{ }^{2}}{\gamma}, \delta_{z} P^{0}$ the pressure gradient along the jet axis $z$, and $C_{1}=\frac{5+\sqrt{7}}{18} \sqrt{\frac{24}{\sqrt{7}-1}}$. When $v_{-}^{*}<0$, the disturbance is not only convected away from the junction but it also travels backwards. The transition from a convectively unstable jet $\left(v_{-}^{*}>0\right)$ to an absolutely unstable jet $\left(v_{-}^{*}<0\right)$ occurs when surface tension equals the critical value

$$
\gamma_{\mathrm{c}}=\frac{-\delta_{z} P^{0} R_{\mathrm{c}}^{2} x^{3} E(x, \lambda)}{C_{1} F(x, \lambda)}
$$

Considering the flow conditions, the fluid properties and the device geometry parameters reported above, we can predict from eqn (4) that for surface tensions higher than $\gamma_{\mathrm{c}} \simeq 0.1 \mathrm{mN} \mathrm{m}^{-1}$, the jet is no longer stable and droplet breakup occurs immediately at the junction.

\section{Acknowledgements}

We are pleased to thank all the members of the Optonanofluidics research team for interesting discussions. This work was supported by EPSRC grants EP/I013342/1 and EP/G00465X/1.

\section{References}

1 R. Seemann, M. Brinkmann, T. Pfohl and S. Herminghaus, Rep. Prog. Phys., 2012, 75, 016601.

2 W. Lee, L. M. Walker and S. L. Anna, Phys. Fluids, 2009, 21, 032103.

3 Y. Song, A. Sauret and H. C. Shum, Biomicrofluidics, 2013, 7, 061301.

4 A. Ashkin, Proc. Natl. Acad. Sci. U. S. A., 1997, 94, 4853-4860. 5 A. Ward, M. Berry, P. Ash, D. Woods and C. Bain, Central Laser Facility-Annual Reports, 2006, 2007, 199-201.

6 A. D. Ward, M. G. Berry, C. D. Mellor and C. D. Bain, Chem. Commun., 2006, 4515-4517.

7 D. A. Woods, C. D. Mellor, J. M. Taylor, C. D. Bain and A. D. Ward, Soft Matter, 2011, 7, 2517-2520.
8 I. Ziemecka, V. van Steijn, G. J. Koper, M. T. Kreutzer and J. H. van Esch, Soft Matter, 2011, 7, 9878-9880.

9 A. Sauret, C. Spandagos and H. C. Shum, Lab Chip, 2012, 12, 3380-3386.

10 K. Tang and A. Gomez, J. Aerosol Sci., 1994, 25, 1237-1249.

11 H. C. Shum, A. Sauret, A. Fernandez-Nieves, H. A. Stone and D. A. Weitz, Phys. Fluids, 2010, 22, 082002.

12 S. D. Geschiere, I. Ziemecka, V. van Steijn, G. J. Koper, J. H. van Esch and M. T. Kreutzer, Biomicrofluidics, 2012, 6, 022007.

13 P. Guillot, A. Colin and A. Ajdari, Phys. Rev. E: Stat., Nonlinear, Soft Matter Phys., 2008, 78, 016307.

14 M. Hashimoto, P. Garstecki, H. A. Stone and G. M. Whitesides, Soft Matter, 2008, 4, 1403-1413.

15 D. Khakhar and J. Ottino, Int. J. Multiphase Flow, 1987, 13, 71-86.

16 P. Janssen, H. Meijer and P. Anderson, Phys. Fluids, 2012, 24, 012102.

17 S. Tomotika, Proc. R. Soc. A, 1935, 150, 322-337.

18 M. K. Mulligan and J. P. Rothstein, Phys. Fluids, 2011, 23, 022004.

19 O. Ghosh and C. A. Miller, J. Phys. Chem., 1987, 91, 45284535.

20 R. Aveyard, B. P. Binks, S. Clark and J. Mead, J. Chem. Soc., Faraday Trans. 1, 1986, 82, 125-142.

21 R. Aveyard, B. P. Binks, T. A. Lawless and J. Mead, Can. J. Chem., 1988, 66, 3031-3037.

22 B. P. Binks, P. D. Fletcher and D. J. Taylor, Langmuir, 1997, 13, 7030-7038.

23 J. Tan, J. Xu, S. Li and G. Luo, Chem. Eng. J., 2008, 136, 306311.

24 G. Bolognesi, Optical studies of micron-scale flows, Lap Lambert Academic Publishing, Saarbrucken, Germany, 2012.

25 J.-C. Baret, F. Kleinschmidt, A. El Harrak and A. D. Griffiths, Langmuir, 2009, 25, 6088-6093.

26 P. Janssen, A. Vananroye, P. Van Puyvelde, P. Moldenaers and P. Anderson, J. Rheol., 2010, 54, 1047-1060.

27 A. Vananroye, P. Van Puyvelde and P. Moldenaers, J. Rheol., 2007, 51, 139-153.

28 V. Sibillo, G. Pasquariello, M. Simeone, V. Cristini and S. Guido, Phys. Rev. Lett., 2006, 97, 054502.

29 T. Fu, Y. Wu, Y. Ma and H. Z. Li, Chem. Eng. Sci., 2012, 84, 207-217.

30 L. Mandelstam, Ann. Phys., 1913, 346, 609-624.

31 D. G. Aarts, M. Schmidt and H. N. Lekkerkerker, Science, 2004, 304, 847-850.

32 P. Guillot, A. Colin, A. S. Utada and A. Ajdari, Phys. Rev. Lett., 2007, 99, 104502. 